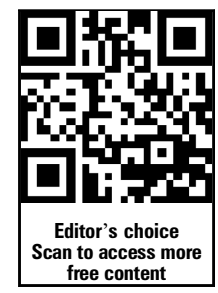

\title{
A crossover randomised controlled trial of oral mandibular advancement devices for obstructive sleep apnoea-hypopnoea (TOMADO)
}

\author{
Timothy G Quinnell, ${ }^{1}$ Maxine Bennett, ${ }^{2}$ Jake Jordan, ${ }^{3}$ Abigail L Clutterbuck-James, ${ }^{1}$ \\ Michael G Davies, ${ }^{1}$ Ian E Smith, ${ }^{1}$ Nicholas Oscroft, ${ }^{1}$ Marcus A Pittman, ${ }^{1}$ \\ Malcolm Cameron, ${ }^{4}$ Rebecca Chadwick, ${ }^{1}$ Mary J Morrell, ${ }^{5}$ Matthew J Glover, ${ }^{3}$ \\ Julia A Fox-Rushby, ${ }^{3}$ Linda D Sharples ${ }^{1,2,6}$
}

\begin{abstract}
- Additional material is published online only. To view please visit the journal online (http://dx.doi.org/10.1136/ thoraxjnl-2014-205464)

${ }^{1}$ Respiratory Support and Sleep Centre, Papworth Hospital NHS Foundation Trust, Cambridge, UK

${ }^{2}$ Medical Research Council Biostatistics Unit, Institute of Public Health, University Forvie Site, Cambridge, UK ${ }^{3}$ Health Economics Research Group, Brunel University, Uxbridge, Middlesex, UK ${ }^{4}$ Department of Oral and Maxillofacial Surgery, Addenbrooke's NHS Foundation Trust, Cambridge, UK

${ }^{5}$ National Heart \& Lung Institute, Royal Brompton Campus, Imperial College, London, UK

${ }^{6}$ Clinical Trials Research Unit, University of Leeds, Leeds, UK
\end{abstract}

\section{Correspondence to} Dr Timothy George Quinnell, Respiratory Support and Sleep Centre, Papworth Hospital NHS Foundation Trust, Papworth Everard, Cambridge CB23 3RE, UK Tim.Quinnell@papworth.nhs.uk

Received 24 March 2014 Revised 6 June 2014 Accepted 25 June 2014 Published Online First 17 July 2014

\section{ABSTRACT}

Rationale Mandibular advancement devices (MADs) are used to treat obstructive sleep apnoea-hypopnoea syndrome (OSAHS) but evidence is lacking regarding their clinical and cost-effectiveness in less severe disease. Objectives To compare clinical- and cost-effectiveness of a range of MADs against no treatment in mild to moderate OSAHS.

Measurements and methods This open-label, randomised, controlled, crossover trial was undertaken at a UK sleep centre. Adults with Apnoea-Hypopnoea Index (AHI) $5-<30 / h$ and Epworth Sleepiness Scale (ESS) score $\geq 9$ underwent 6 weeks of treatment with three nonadjustable MADs: self-moulded (SleepPro 1; SP1); semi-bespoke (SleepPro 2; SP2); fully-bespoke MAD (bMAD); and 4 weeks no treatment. Primary outcome was $\mathrm{AHI}$ scored by a polysomnographer blinded to treatment. Secondary outcomes included ESS, quality of life, resource use and cost.

Main results 90 patients were randomised and 83 were analysed. All devices reduced $\mathrm{AHI}$ compared with no treatment by $26 \%(95 \% \mathrm{Cl} 11 \%$ to $38 \%, p=0.001)$ for SP1, 33\% (95\% Cl $24 \%$ to $41 \%)$ for SP2 and $36 \%$ (95\% Cl $24 \%$ to $45 \%, p<0.001)$ for bMAD. ESS was $1.51(95 \% \mathrm{Cl} 0.73$ to $2.29, \mathrm{p}<0.001, \mathrm{SP} 1)$ to 2.37 (95\% Cl 1.53 to $3.22, p<0.001$, bMAD) lower than no treatment $(p<0.001$ for all). Compliance was lower for SP1, which was the least preferred treatment at trial exit All devices were cost-effective compared with no treatment at a $£ 20$ 000/quality-adjusted life year (QALY) threshold. SP2 was the most cost-effective up to f39 800/QALY.

Conclusions Non-adjustable MADs achieve clinically important improvements in mild to moderate OSAHS and are cost-effective. Of those trialled, the semi-bespoke MAD is an appropriate first choice.

Trial registration number ISRCTN02309506.

\section{INTRODUCTION}

Obstructive sleep apnoea-hypopnoea (OSAH) involves repeated collapse of the pharyngeal airway during sleep, causing oxygen desaturations and brief arousals. OSAH syndrome (OSAHS) incorporates excessive daytime sleepiness (EDS), ${ }^{1}$ affecting $2 \%-7 \%$ of adults. ${ }^{2}$

There is a causal link with hypertension ${ }^{3}$ and cardiovascular risk is increased 2.5 -fold, ${ }^{4}$ with a

\section{Key messages}

\section{What is the key question?}

- Are mandibular advancement devices (MADs) clinically- and cost-effective compared with no treatment in mild to moderate obstructive sleep apnoea-hypopnoea syndrome (OSAHS), and does the degree of MAD sophistication influence outcomes?

What is the bottom line?

- Clinical and cost-effectiveness analyses suggest that semi-bespoke non-adjustable devices should be offered as first line treatment for mild OSAHS and as an alternative to CPAP in moderate disease, whereas dentally-fitted bespoke devices should be reserved for those who cannot produce the mould for, or tolerate, a semi-bespoke device; and while adjustable MADs offer some advantages, their precise role and cost-effectiveness still need to be established.

\section{Why read on?}

- This is the first comprehensive randomised controlled trial to evaluate both the clinical and cost-effectiveness of MADs for the treatment of mild to moderate OSAHS; the results and their implications for clinical practice are discussed.

reported 6\% increase in stroke risk per unit increase in Apnoea-Hypopnoea Index (AHI/hour). ${ }^{5}$ Road traffic accident risk is two to three times higher $^{6}$ and health-related quality of life (HRQoL) is impaired. ${ }^{7}$ Healthcare usage is almost doubled in OSAHS, with the main determinants of increased cost being cardiovascular disease and psychoactive medication. $^{8}$

Weight loss sometimes cures OSAHS but CPAP therapy gives immediate control of obstructive events. Improvement in EDS usually follows, with added benefits to driving safety and HRQoL. ${ }^{9}$ Meta-analyses have shown that CPAP reduces mean blood pressure by around $2 \mathrm{~mm} \mathrm{Hg}^{10}$ and observational data have suggested cardiovascular risk reduction. $^{11}$ 
CPAP is recommended by the National Institute for Health and Care Excellence as clinically and cost-effective for moderate to severe OSAHS. ${ }^{12}$ Benefits are less certain in milder disease, although there is some evidence of improvement in functional outcomes and daytime sleepiness. ${ }^{13}{ }^{14}$ Intolerance of CPAP is common, affecting $46 \%-83 \%$ of patients across the disease spectrum. ${ }^{15}$ Effective alternatives to CPAP are therefore needed.

Intraoral mandibular advancement devices (MADs) protrude the mandible and tongue to maintain upper airway patency during sleep. Meta-analyses suggest MADs are beneficial in OSAHS, although trial and device heterogeneity complicate interpretation. ${ }^{16}$ Most comparative studies show CPAP is better at controlling respiratory events but both treatments improve sleepiness equally, possibly due to CPAP intolerance. ${ }^{17}$ MADs are effective compared with sham MADs in reducing AHI and improving sleepiness. However, adverse sham effects may exaggerate treatment benefits of active devices by undermining sleep quality without reducing respiratory events, ${ }^{16}$ although there is evidence to the contrary in more severe OSAHS. ${ }^{18}$ Nonetheless, it is important to compare the effectiveness of MADs with no treatment in milder disease, where the balance of costs and benefits may be more marginal. ${ }^{19}$ The considerable heterogeneity in MAD treatment, caused by variation in device design, production processes and specialist involvement, also results in cost variability. There is therefore a need to explore the clinical and cost-effectiveness of a range of MADs. This study aimed to determine whether:

1. MADs are clinically- and cost-effective compared with no treatment in mild to moderate OSAHS.

2. The degree of MAD sophistication influences outcomes, including cost-effectiveness.

\section{METHODS}

Open-label, four-period, crossover, randomised controlled trial (RCT) comparing three non-adjustable MADs with no treatment.

Full details of trial methodology are included in an online supplement.

\section{Participants}

Patients aged $\geq 18$ years with mild to moderate OSAHS confirmed by respiratory polysomnography (rPSG) (AHI $5-<30 / \mathrm{h}$ ) and symptomatic daytime sleepiness (Epworth Sleepiness Scale (ESS) score $\geq 9$ ) were recruited from Papworth Hospital sleep centre. Newly diagnosed patients not requiring or declining CPAP and existing CPAP intolerant patients were eligible. See online supplement for exclusion criteria.

\section{Procedures}

The three MADs were: thermoplastic 'boil and bite' device (SleepPro 1 (SP1); Meditas, Winchester, UK); semi-bespoke device produced from a patient-moulded dental impression kit (SleepPro 2 (SP2); Meditas); bespoke MAD (bMAD) device fitted and manufactured by National Health Service (NHS) Maxillofacial Team at Addenbrooke's Hospital, Cambridge, UK.

After dental eligibility was confirmed, patients were randomised via telephone by the hospital's R\&D unit using Williams' Latin Squares with allocations generated by computer, using permuted blocks of eight.

Period duration was 6 weeks (4 weeks for no treatment): 2 weeks acclimatisation and 4 weeks treatment. One week's washout followed active treatments. Outcomes were obtained at baseline and at the end of each treatment period.

\section{Outcomes}

Primary outcome was AHI, measured by domiciliary rPSG (Embletta, Embla Systems, Kanata, Ontario, Canada) and scored by a polysomnographer blinded to treatment. Other outcomes included rPSG indices, blood pressure, subjective sleepiness (ESS), sleep-related quality of life (Functional Outcomes of Sleep Questionnaire (FOSQ); Calgary Sleep Apnoea Quality of Life Index (SAQLI)) and generic HRQoL (Short Form 36 (SF36); EuroQol (EQ-5D-3L)). Healthcare usage, road traffic accidents and treatment satisfaction were recorded at the end of each treatment period. Treatment compliance was calculated at the end of each treatment period from a patient-completed diary. Device preference was documented at trial exit.

\section{Statistical analysis}

Calculations indicated 72 patients for $80 \%$ power to detect treatment effects of $1 / 3$ SDs between MADs (two-sided 5\% significance). A total of 90 patients were recruited, allowing 20\% loss to follow-up. All randomised patients were followed up and available results from periods included in analysis irrespective of treatment compliance ('intention to treat').

Mixed effects Poisson regression was used to estimate effects of treatment, period and treatment by period interactions for AHI. Mixed effects logistic regression was used to analyse response (complete (AHI $<5$ ) or partial (AHI $\geq 5$ with $\geq 50 \%$ reduction from baseline)). All other outcomes were analysed using linear mixed models.

Regressions explored the effects on AHI response of baseline AHI, ESS, age, gender, compliance and body mass index (BMI), and BMI changes over time.

\section{Economic analysis}

Using an NHS perspective, device costs, fitting time and other healthcare usage within each treatment period were compared against the costs of no treatment. Patient-specific healthcare resource use data were collected and valued using NHS reference costs, standard unit costs and published literature. ${ }^{20} 21$ HRQoL was measured and valued using the EQ-5D-3L and UK social tariff and converted to quality-adjusted life years (QALYs). ${ }^{22} \mathrm{~A}$ random effects model estimated differences in costs and QALYs for each MAD against control. Bootstrapping was used to construct cost-effectiveness acceptability curves (CEACs) and cost-effectiveness acceptability frontier. The impact of changes in MAD cost, device lifespan and use of SF6D QALYs on net monetary benefit (NMB) was assessed.

\section{RESULTS}

In all, 90 patients were randomised between December 2010 and July 2012 (figure 1). A total of $16(18 \%)$ withdrew from the trial; $7(8 \%)$ of these did not complete any treatment and were excluded from analyses. Two additional patients who withdrew between periods one and two had a failed sleep study but completed secondary outcomes, making $9(10 \%)$ patients who provided no AHI data after baseline. Seven patients withdrew later in the trial. It has been assumed that the data were missing at random for these patients. There were no differences in baseline characteristics between those who completed the trial and those who withdrew, and the pattern of withdrawal did not correspond to any particular MAD (see online supplement). Seven other sleep studies failed, leaving 305 studies (85\% of 360 ) from 81 patients $(90 \%)$ for AHI analysis. For all other outcomes, $314(87 \%)$ measurements and 83 (92\%) patients were 


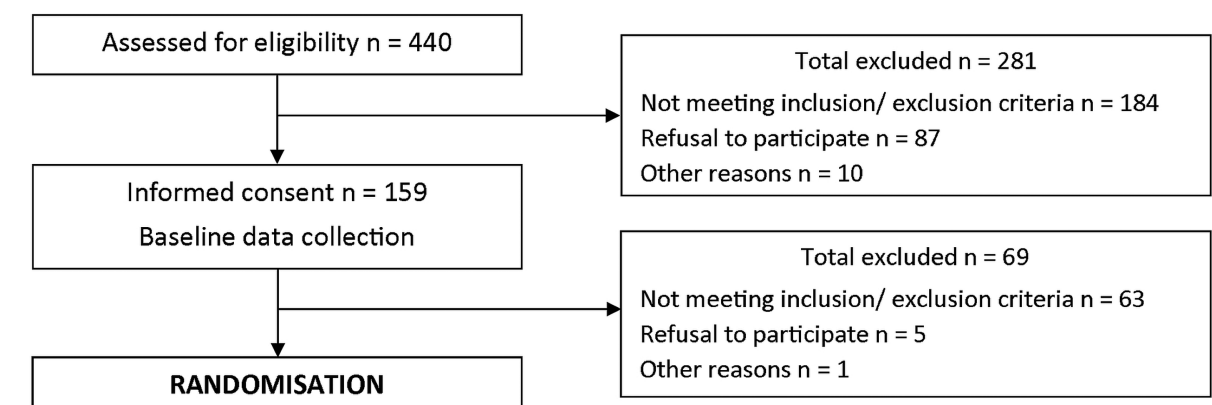

Allocated to intervention $\mathrm{n}=90$

Received allocated intervention $n=90$

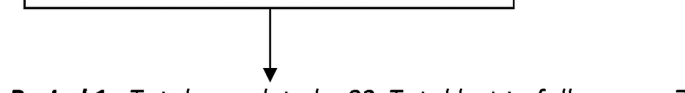

Period 1 - Total completed $=83$, Total lost to follow-up $=7$

\begin{tabular}{|c|c|c|c|}
\hline No treatment $n=\mathbf{2 1}$ & SP1 $n=21$ & $S P 2 n=21$ & bMAD $n=20$ \\
\hline Lost to follow-up $n=1$ & Lost to follow-up $n=2$ & Lost to follow-up $n=1$ & Lost to follow-up $n=3$ \\
\hline \multirow[t]{2}{*}{$1=$ Consent withdrawn } & $1=\mathrm{AE}-$ patient decision & $1=$ Consent withdrawn & $1=\mathrm{AE}-$ clinical decision \\
\hline & $1=$ Consent withdrawn & & $\begin{array}{l}1=\mathrm{AE}-\text { patient decision } \\
1=\text { Consent withdrawn }\end{array}$ \\
\hline
\end{tabular}

Period 2 - Total completed $=80$, Total lost to follow-up $=3$ (cumulative $=10$ )

No treatment $n=21$
Lost to follow-up $n=0$

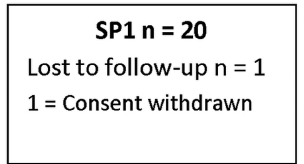

SP2 $\mathbf{n}=\mathbf{2 0}$
Lost to follow-up $n=1$
$1=$ Other

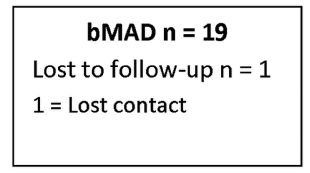

Period 3 - Total completed $=77$, Total lost to follow-up $=3$ (cumulative $=13$ )

\begin{tabular}{|c|c|c|}
\hline $\begin{array}{l}\text { No treatment } n=21 \\
\text { Lost to follow-up } n=0\end{array}$ & $\begin{array}{c}\text { SP1 } n=20 \\
\text { Lost to follow-up } n=0\end{array}$ & $\begin{array}{l}\quad \text { SP2 } \mathbf{n}=18 \\
\text { Lost to follow-up } n=2 \\
1=\text { Consent withdrawn } \\
1 \text { = Other }\end{array}$ \\
\hline
\end{tabular}

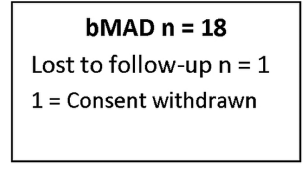

Period 4 - Total completed $=74$, Total lost to follow-up $=3$ (cumulative $=16$ )

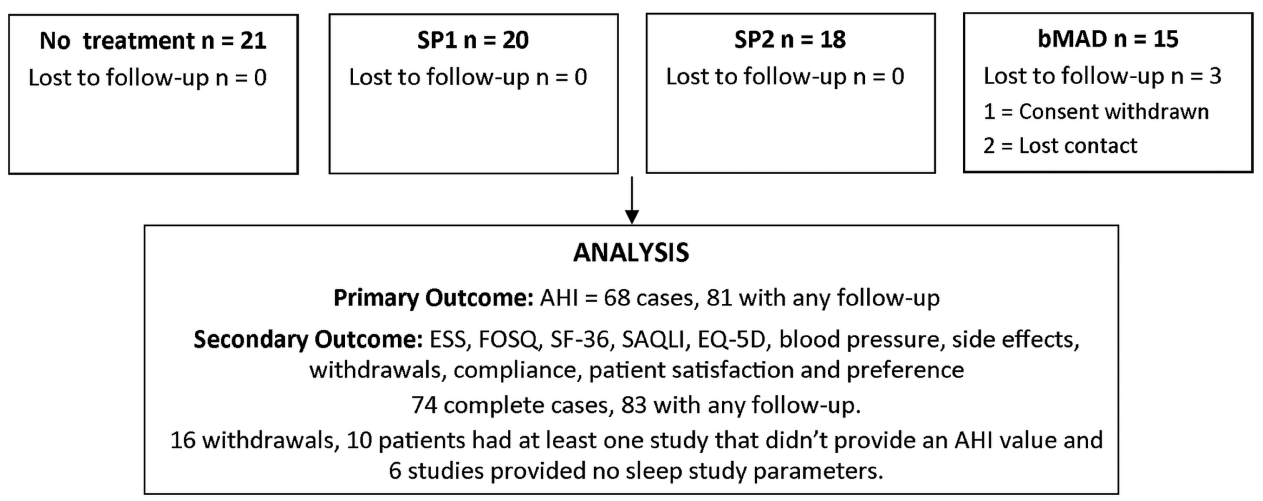

Figure 1 Flow of patients through the trial.

available for analyses. Baseline characteristics for complete cases and patients with missing data were similar.

Baseline characteristics for randomised patients are given in table 1.

Although 12 patients had baseline ESS below 9, they were eligible based on their ESS being 9 or more at screening. One patient was erroneously randomised following a screening ESS of 8 (ESS $=10$ at baseline). Three patients were randomised on the basis of more sensitive electroencephalographicallyguided AHI scoring. When later rescored according to rPSG criteria, the AHI was below 5. There were no significant period-by-treatment interactions and, for AHI and ESS, no period effects. Complete case results were almost identical to main analysis of cases with at least one measurement, so only the latter is reported.

All three MADs significantly decreased the AHI against no treatment by $26 \%$ (95\% CI $11 \%$ to $38 \%$ ) for the SP1, 33\% (95\% CI $24 \%$ to $41 \%$ ) for the SP2 and 36\% (95\% CI $24 \%$ to $45 \%$ ) for the bMAD (table 2, figure $2 \mathrm{~A}$ ).

A similar effect was found for all devices against no treatment for $4 \%$ oxygen desaturation index (4\% ODI). There were no significant differences between devices for either AHI or 4\% ODI. Compared with no treatment, patients spent significantly less time with nocturnal oxygen saturation $<90 \%$ when using SP2 and bMAD (SP2 vs no treatment $\mathrm{p}=0.040$ and bMAD vs no treatment $\mathrm{p}<0.001$, respectively), and there were some 
Table 1 Baseline characteristics

\begin{tabular}{|c|c|c|c|}
\hline Unit/category & Total $(\mathrm{N}=90)$ & Min & Max \\
\hline \multicolumn{4}{|l|}{ Demographic information } \\
\hline \multicolumn{4}{|l|}{ Gender } \\
\hline Male & $72(80 \%)$ & & \\
\hline Female & $18(20 \%)$ & & \\
\hline \multicolumn{4}{|l|}{ Age at randomisation } \\
\hline Years & 50.9 (11.6) & 26.1 & 79.6 \\
\hline \multicolumn{4}{|l|}{ BMI } \\
\hline $\mathrm{Kg} / \mathrm{m}^{2}$ & $30.6(27.9-35.1)$ & 23.9 & 54.5 \\
\hline \multicolumn{4}{|l|}{ Smoking history } \\
\hline Non-smoker & $44(49 \%)$ & & \\
\hline Ex-smoker & $39(43 \%)$ & & \\
\hline Smoker & $7(8 \%)$ & & \\
\hline \multicolumn{4}{|l|}{ Clinical history } \\
\hline Previous CPAP & $4(4 \%)$ & & \\
\hline Asthma & $14(16 \%)$ & & \\
\hline \multicolumn{4}{|l|}{ Diabetes } \\
\hline Type I & $1(1 \%)$ & & \\
\hline Type II & $7(8 \%)$ & & \\
\hline \multicolumn{4}{|l|}{ Cardiovascular disease } \\
\hline Previous stroke & $2(2 \%)$ & & \\
\hline Previous TIA & $1(1 \%)$ & & \\
\hline Ischaemic heart disease & $5(6 \%)$ & & \\
\hline Hypertension & $23(26 \%)$ & & \\
\hline \multicolumn{4}{|l|}{ Sleep study } \\
\hline \multicolumn{4}{|l|}{ Apnoea-Hypopnoea Index } \\
\hline Events per hour & $13.8(6.2)$ & 2.9 & 27.7 \\
\hline Missing* & 1 & & \\
\hline \multicolumn{4}{|l|}{ Oxygen Desaturation Index } \\
\hline Events per hour & $9.8(5.2)$ & 0.6 & 22 \\
\hline \multicolumn{4}{|l|}{ Minimum $\mathrm{SpO}_{2}$} \\
\hline Per cent & $83.7(4.7)$ & 71 & 91 \\
\hline Missing* & 2 & & \\
\hline \multicolumn{4}{|l|}{ Mean $\mathrm{SpO}_{2}$} \\
\hline Per cent & $94.2(1.3)$ & 89.8 & 97.7 \\
\hline Missing* & 1 & & \\
\hline \multicolumn{4}{|c|}{ Time $<90 \%$ of nocturnal $\mathrm{SpO}_{2}$} \\
\hline Minutes & $8.3(2.9-24.8)$ & 0 & 315.4 \\
\hline Missing* & 1 & & \\
\hline \multicolumn{4}{|c|}{ Epworth Sleepiness Scale (ESS) } \\
\hline \multicolumn{4}{|l|}{ ESS } \\
\hline Unit score & $11.9(3.5)$ & 3 & 20 \\
\hline
\end{tabular}

differences between MADs (bMAD vs SP2, uncorrected for multiple testing $p=0.037$; bMAD vs SP1, uncorrected multiple testing $p=0.006$ ). The $b M A D$ had a significant effect on minimum oxygen saturation compared with no treatment and the other devices (see online supplement).

Complete response (AHI $<5)$ or partial response $(\geq 50 \%$ reduction in AHI from baseline but $\mathrm{AHI} \geq 5$ ) to treatment was observed in 17 of 76 (22\%) cases after no treatment, and 29/77 (38\%), 38/78 (49\%) and 33/74 (45\%) patients after SP1, SP2 and bMAD, respectively ( $p=0.0006$ for all vs no treatment; see online supplement). Response was significantly associated with baseline BMI (OR $0.89 / \mathrm{kg} / \mathrm{m}^{2}, 95 \%$ CI 0.80 to $0.98, \mathrm{p}=0.014$ ) and contemporaneous BMI (OR $0.88 / \mathrm{kg} / \mathrm{m}^{2}, 95 \%$ CI 0.80 to $0 \cdot 96, \mathrm{p}=0 \cdot 007)$. There was a weak association with protrusion
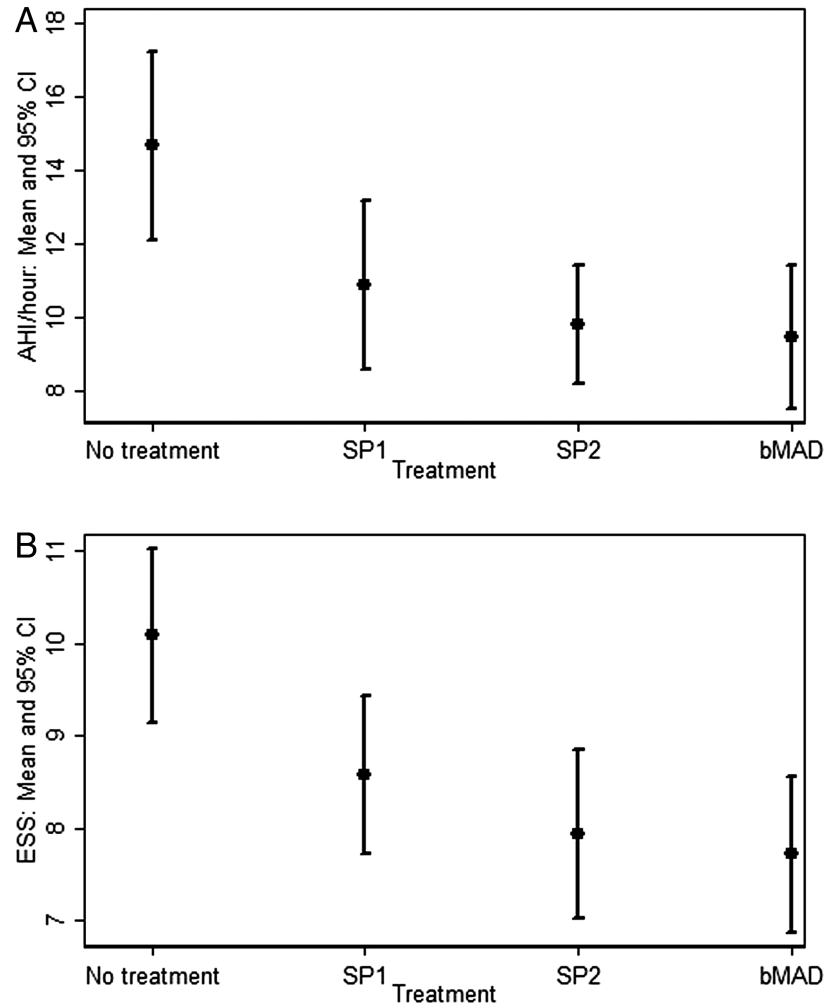

Figure 2 (A) Mean Apnoea-Hypopnoea Index (AHI) and (B) mean Epworth Sleepiness Scale (ESS) score (with 95\% Cls for each treatment) from the Poisson mixed effects model.

(OR 1.03 per \% maximal protrusion, 95\% CI 1.00 to 1.05 , $\mathrm{p}=0.034)$, although there was no association between \% protrusion and AHI as a continuous variable (HR 0.997 (0.991 to 1.001), $\mathrm{p}=0.206$ ). Baseline AHI, ESS, gender, age and compliance were not associated with treatment response.

Median (quartiles) number of nights (of 28) that SP1, SP2 and bMAD were used was $25(17,28), 27(23,28)$ and $26(23$, $28)$, respectively. Mean (SD) nightly use of each device was 4.4 (2.4), 5.7 (2.0) and 5.7 (2.0) hours, respectively $(p<0.001$ for both SP2 and bMAD vs SP1). Patients stopped treatment early during 14 of 81 (17\%) SP1, four of 78 (5\%) SP2 and six of 76 (8\%) bMAD periods $(\mathrm{p}=0.034)$.

All MADs decreased ESS significantly compared with no treatment, by 1.51 units (95\% CI 0.73 to 2.29 ) for the SP1, 2.15 units (95\% CI 1.31 to 2.99) for the SP2 and 2.37 units (95\% CI 1.53 to 3.22) for the bMAD (table 2, figure 2B). Based on Bonferroni corrected comparisons, there were no significant differences between devices.

Compared with the no treatment arm, total FOSQ score was significantly higher (better) by 0.50 (95\% CI 0.08 to 0.92 , $\mathrm{p}=0.018)$ for SP1, $1.10(95 \%$ CI 0.65 to $1.55, \mathrm{p}<0.001)$ for SP2 and $1.31(95 \%$ CI 0.84 to $1.78, \mathrm{p}<0.001)$ for bMAD. Total SAQLI score was improved in a similar pattern compared with no treatment: 0.27 (95\% CI 0.07 to $0.48, \mathrm{p}=0.008)$ for the SP1, $0.62(95 \%$ CI 0.38 to $0.86, \mathrm{p}<0.001)$ for the SP2 and $0.65(95 \%$ CI 0.41 to $0.90, p<0.001)$ for the bMAD. The SP2 and bMAD FOSQ and SAQLI scores were significantly higher (better) than the SP1 (uncorrected $\mathrm{p} \leq 0.01$ ). While most of the SF36 dimensions did not show differences between MADs, there were important differences in the Vitality Scores for SP2 and bMAD against no treatment (see online supplement). 
Table 2 Summary of results from mixed effects models for AHI $(n=81)$ and ESS $(n=83)$

\begin{tabular}{|c|c|c|c|c|c|}
\hline & Mean (SD) & Coefficient & $95 \% \mathrm{Cl}$ & $\mathrm{p}$ Value & Global $p$ value \\
\hline \multicolumn{6}{|l|}{$A H I(n=81)$} \\
\hline Constant & & 14.22 & (11.66 to 17.34$)$ & $<0.001$ & \\
\hline \multicolumn{6}{|c|}{ Relative AHI compared with no treatment } \\
\hline No treatment & $14.6(10.5)$ & - & - & - & $<0.001$ \\
\hline SP1 & $10.8(9.5)$ & 0.74 & (0.62 to 0.89 ) & 0.001 & \\
\hline SP2 & $9.7(8.9)$ & 0.67 & (0.59 to 0.76$)$ & $<0.001$ & \\
\hline bMAD & $9.5(8.4)$ & 0.64 & (0.55 to 0.76$)$ & $<0.001$ & \\
\hline \multicolumn{6}{|l|}{ ESS $(n=83)$} \\
\hline Constant & & 10.65 & (9.64 to 11.66$)$ & $<0.001$ & \\
\hline \multicolumn{6}{|c|}{ Difference in ESS compared with no treatment } \\
\hline No treatment & $10.1(4.3)$ & - & - & - & $<0.001$ \\
\hline SP1 & $8.5(4.0)$ & -1.51 & $(-2.29$ to -0.73$)$ & $<0.001$ & \\
\hline SP2 & $8.0(4.1)$ & -2.15 & $(-2.99$ to -1.31$)$ & $<0.001$ & \\
\hline bMAD & $7.7(3.8)$ & -2.37 & $(-3.22$ to -1.53$)$ & $<0.001$ & \\
\hline
\end{tabular}

AHI, Apnoea-Hypopnoea Index; bMAD, bespoke mandibular advancement device; ESS, Epworth Sleepiness Scale.

Mean (SD) mandibular protrusion for the SP1 was greater than for the other two devices (SP1, 62.6\% (22.1); SP2, 51.7\% (26.4), uncorrected $\mathrm{p}<0.001$; bMAD, 55.2\% (19.7), uncorrected $\mathrm{p}=0.012)$. Patients found the SP1 less comfortable and were less satisfied with it than the SP2 and bMAD (see online supplement). The SP1 was more likely to fall out or be removed.

Of 74 trial completers, $30(41 \%)$ ranked the bMAD highest in terms of preference and $23(31 \%)$ ranked it second (see online supplement). SP2 was ranked highest by 22 (30\%) and second by $34(46 \%)$ patients, while $10(14 \%)$ favoured no treatment. After the trial, 56 of $74(76 \%)$ completers continued treatment with their preferred MAD and $4(5 \%)$ others chose treatment with the MAD that achieved the best AHI.

There were four serious adverse events during the trial. Minor adverse events were experienced by 86 (96\%) patients. Most common were mouth problems/discomfort (83, 92\%) and excess salivation $(48,53 \%)$ with SP2 performing best for both (see online supplement).

All devices were cost-effective compared with no treatment at a willingness-to-pay (WTP) of $£ 20000 /$ QALY, based on mean costs and QALYs. SP2 achieved the highest NMB at $£ 33$ per 4 weeks (table 3 ).

On average, the SP1 and SP2 point estimates were associated with more QALYs and lower mean costs compared with no treatment, although QALY differences between devices were small and non-significant. These results are robust to: changes in a device's price and lifespan; increasing the WTP per QALY to $£ 30000$; and using only complete case analysis. When the bMAD price exceeds $£ 525$ or average lifespan falls below 14 months, it no longer has a positive NMB. The CEAC (figure $3 \mathrm{~A}$ ) and NMB (figure 3B) show SP2 to be most costeffective up to a WTP of $£ 39800$ / QALY, at which point bMAD supersedes it (39\% likelihood of being cost-effective vs 35\% for the SP2). Below a WTP of $£ 5000 / \mathrm{QALY}$, only SP2 is more costeffective than no treatment. The finding that SP2 was the most cost-effective option was strengthened considerably when using SF6D QALYs.

\section{DISCUSSION}

This trial showed that in mild to moderate OSAHS, nonadjustable MADs improve objective and subjective health outcomes over no treatment. Additional improvements diminished with increasing MAD sophistication but the consistent results across outcomes suggest genuine effects. All devices were cost-effective against no treatment based on the point estimates of costs and QALYs. However, differences in EQ-5D-3L results between devices were small and non-significant, although significant using SF6D QALYs. Probabilistic analysis, accounting for uncertainty in costs and QALYs, showed SP2 was the most costeffective up to a WTP of $£ 39800 /$ QALY. Above this WTP, bMAD appeared most cost-effective in the short term, although not using SF6D QALYs.

All MADs reduced AHI to between $64 \%$ and $75 \%$ of no treatment AHI, which is modest. Pneumatic splinting of CPAP efficiently controls multilevel pharyngeal collapse. The mechanisms of action of MADs are more complex, probably involving airway stiffening, splinting and enlargement. ${ }^{23}$ These factors and level of obstruction vary between patients, impacting MAD efficacy. Greater AHI effects have been reported ${ }^{24} 25$ but methodological and device heterogeneity complicate interpretation of the comparisons. Many studies also include patients with more severe disease at baseline, ${ }^{24} 26$ which gives more potential for useful treatments to show an effect. The focus of the trial of oral mandibular advancement devices for obstructive sleep apnoea-hypopnoea (TOMADO) was on milder disease, where a no treatment control was particularly relevant. The RCT most comparable with TOMADO, which used a maximally titrated adjustable MAD in mild to moderate OSAHS, reported a mean $\mathrm{AHI}$ of $67 \%$ against placebo tablet, which is consistent with our results. $^{9}$

Reduction of AHI can be proportional to mandibular protrusion. ${ }^{27}$ Mean (SD) protrusion in this trial (4.8 (2.5) (SP2) to 5.7 (2.1) (SP1) $\mathrm{mm}(52.5 \quad(27.8) \%$ to $63.4 \quad(22.6) \%$ maximal advancement)) was lower than others have achieved, ${ }^{18} 28$ often using adjustable MADs. ${ }^{9} 17242629$ However, greater protrusion in those trials did not always achieve greater AHI reduction than TOMADO. ${ }^{9} 18$ The results from this trial did not demonstrate a convincing association between protrusion and AHI. There is also evidence that maximal protrusion may not be necessary in milder OSAHS. ${ }^{30}$

All MADs studied were associated with a statistically significant improvement in ESS. The SP1 improvement was of borderline clinical significance compared with no treatment. The ESS 
Table 3 Comparison of costs and QALYs from devices against control

\begin{tabular}{|c|c|c|c|c|c|c|c|c|}
\hline \multirow[b]{2}{*}{ Cost component (f) } & \multicolumn{8}{|c|}{ Intervention } \\
\hline & \multicolumn{2}{|c|}{$\begin{array}{l}\text { No treatment } \\
n=78\end{array}$} & \multicolumn{2}{|l|}{$\begin{array}{l}S P 1 \\
n=81\end{array}$} & \multicolumn{2}{|l|}{$\begin{array}{l}S P 2 \\
n=78\end{array}$} & \multicolumn{2}{|l|}{$\begin{array}{l}\text { bMAD } \\
n=77\end{array}$} \\
\hline Device (fixed) & \multicolumn{2}{|l|}{-} & \multicolumn{2}{|l|}{$£ 21$} & \multicolumn{2}{|l|}{ f128 } & \multicolumn{2}{|l|}{ £350 } \\
\hline Measurement for device (fixed) & \multicolumn{2}{|l|}{-} & \multicolumn{2}{|l|}{-} & \multicolumn{2}{|l|}{-} & \multicolumn{2}{|l|}{ f110.37 } \\
\hline Fitting of device (fixed) & \multicolumn{2}{|l|}{-} & \multicolumn{2}{|l|}{-} & \multicolumn{2}{|l|}{-} & \multicolumn{2}{|l|}{ £92.04 } \\
\hline Additional visit if required (average across all patients) & \multicolumn{2}{|l|}{-} & \multicolumn{2}{|l|}{-} & \multicolumn{2}{|l|}{-} & \multicolumn{2}{|l|}{ f5.98 } \\
\hline Subtotal & \multicolumn{2}{|l|}{-} & \multicolumn{2}{|l|}{$\mathrm{f} 21$} & \multicolumn{2}{|l|}{ f128 } & \multicolumn{2}{|l|}{$£ 350$} \\
\hline Device lifespan (months) (fixed) & \multicolumn{2}{|l|}{-} & \multicolumn{2}{|l|}{12} & \multicolumn{2}{|l|}{12} & \multicolumn{2}{|l|}{18} \\
\hline Cost of intervention subtotal pro rata (4 weeks) (fixed)* & \multicolumn{2}{|l|}{-} & \multicolumn{2}{|l|}{ f1.62 } & \multicolumn{2}{|l|}{ £9.85 } & \multicolumn{2}{|l|}{$\mathrm{f} 28.64$} \\
\hline Summary of costs ( $f$ ) & Mean & SE & Mean & SE & Mean & SE & Mean & SE \\
\hline Resource use cost (4 weeks) Mean (SD) $\dagger$ & f78.50 & £19.97 & f73.02 & f10.47 & £53.58 & $\mathrm{f} 8.05$ & f76.25 & $\mathrm{f} 24.40$ \\
\hline Total cost (4 weeks) Mean (SD)† & f78.50 & £19.97 & £74.64 & f10.47 & f63.43 & f8.05 & f104.89 & $\mathrm{f} 24.39$ \\
\hline Total cost difference intervention vs control & - & - & $-\mathrm{f} 3.87$ & f21.38 & $-f 15.08$ & $\mathrm{f} 20.62$ & £26.39 & £27.94 \\
\hline \multicolumn{9}{|l|}{ Health-related quality of life measure } \\
\hline EQ-5D-3L Utility score & 0.85 & 0.2 & 0.86 & 0.2 & 0.86 & 0.23 & 0.87 & 0.19 \\
\hline EQ-5D-3L QALY score (4-week trial period)‡ & 0.0649 & 0.0017 & 0.0658 & 0.0017 & 0.0658 & 0.0019 & 0.0667 & 0.0017 \\
\hline QALY score difference intervention vs control & - & - & 0.00094 & 0.00105 & 0.00088 & 0.00123 & 0.00177 & 0.00147 \\
\hline Cost effectiveness measure & & & & & & & & \\
\hline Incremental Cost-Effectiveness Ratio & - & & $-£ 4093$ & & $-\mathrm{f17} 104$ & & f14 876 & \\
\hline Net monetary benefit (willingness-to-pay=f20 000) & - & & $\mathrm{f} 23$ & & $\mathrm{f} 33$ & & f9 & \\
\hline
\end{tabular}

${ }^{*}$ Device and fitting costs are pro rata to be comparable over the 4-week trial period.

tResource use and total costs by intervention estimated using a mixed effects model controlling for baseline data. All costs in 2011/2012 (f).

$\ddagger$ QALY scores calculated using area under the curve method to represent the true QALY score for the 4-week intervention period and to be consistent with the costs presented.

bMAD, bespoke mandibular advancement device; QALY, quality-adjusted life year.

effects for the SP2 and bMAD were greater and similar to some of those reported for CPAP in OSAHS. ${ }^{17}$ Placebo effects cannot be ignored. A recent study attributed up to $29 \%$ of ESS response to expectation of benefit from high CPAP compliance $;{ }^{31}$ but the associated objective AHI reductions in this study suggest real effects.

Patient-reported MAD compliance was good, and the reliability of subjective measurement has been objectively demonstrated. ${ }^{32}$ Compliance was lower for SP1, which was less well tolerated and often fell out or was removed. Poor retention of a non-bMAD has been related to inferior compliance, ${ }^{25}$ while superior MAD compliance is considered key to matching CPAP health outcomes. ${ }^{17}$ The SP1's unpopularity at trial exit and poorer in-trial compliance suggest there are significant obstacles to its longer term effectiveness.

This relatively large study used an efficient design to estimate the short-term effectiveness of MADs on the most relevant outcomes in OSAHS: AHI and EDS. The results were clear, unambiguously significant and robust to assumptions for incomplete data. Seven patients failed to complete any treatment and two dropped out after one period. We therefore estimate that $10 \%$ of patients eligible for a MAD will prove intolerant. Another 8\% may become intolerant later. This highlights the need for longer term data regarding MAD usage. Current ongoing follow-up of trial patients will eventually assess longer term device durability and compliance but until this information becomes available, modelling of long-term outcomes must be relied upon.

This study was conducted at a specialist centre, potentially limiting generalisability. However, participants were recruited from our usual clinic population, mostly referred from primary care. The SP1 and SP2 are available in many countries and similar to other thermoplastic and 'semi-bespoke' MADs. The bMAD was similar in design to other available monobloc devices. It was fitted and manufactured by a hospital maxillofacial laboratory, but using skills, materials and facilities common to dental sleep services.

Although the aim for the bMAD was at least 50\% maximal protrusion, this was often lower and similar to that achieved independently by patients with the other devices. This reflects the pragmatic nature of the trial, making its findings more applicable to the wider NHS. Including an adjustable MAD may have achieved greater protrusion and would have extended this effectiveness evaluation. However, the need to adequately cover the range of non-adjustable MADs available to NHS sleep services precluded this. Although they are more costly, adjustable MADs are increasingly recommended. ${ }^{33}{ }^{34}$ Titration is thought to optimise protrusion and tolerance ${ }^{35}$ but their superiority remains unproven. A large retrospective review of mild to severe OSAHS patients fitted with a bMAD reported slightly greater AHI effects for an adjustable MAD compared with a non-adjustable device. However, differences were often not statistically significant and clinical significance was doubtful given that ESS reductions were no different. ${ }^{34}$ The inconsistent evidence regarding greater protrusion in milder disease has already been discussed, and the high reported compliance with our bespoke devices leaves little room for improvement. Nonetheless, the increasing popularity of more expensive adjustable MADs means rigorous prospective comparisons should be a priority.

In the short term, MADs achieve clinically significant improvements in mild to moderate OSAHS and appear costeffective at $£ 20000 / Q A L Y$ compared with no treatment. Inferior tolerance and retention of the SP1 device may limit its effectiveness. Minor differences between the two more sophisticated devices suggest that a semi-bespoke non-adjustable MAD could be a practical and efficient first choice in most patients. A 

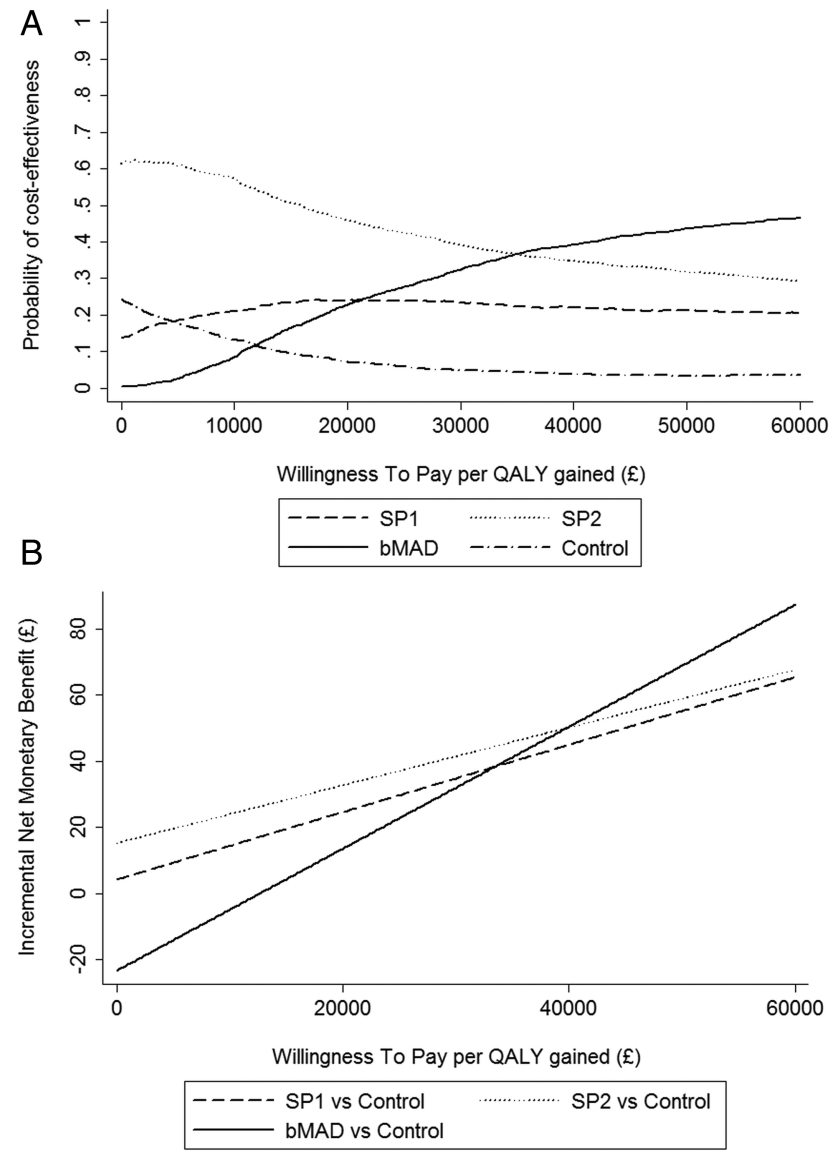

Figure 3 (A) Cost-effectiveness acceptability curves (CEACs) of each device compared with all alternatives and (B) net monetary benefit (NMB) for each device compared with no treatment (control). The CEAC is derived from the joint distribution of incremental costs and incremental quality-adjusted life years (QALYs) and shows the uncertainty associated with both. The curve shows the probability that a specific treatment (SP1, SP2, bespoke mandibular advancement device (bMAD) or no treatment) will be cost-effective compared with all the other alternatives assessed for a specific amount that a service provider is willing to pay per QALY gained. The NMB is the monetary value of the devices incremental health effect (ie, the willingness-to-pay for a QALY gain $\times$ total QALYs gained minus the incremental cost). It is measured over the 4-week period of the trial. A higher NMB is associated with a more cost-effective treatment.

bespoke device may be necessary for those patients who require dental help with production and fitting of a MAD, or where there are concerns regarding dental eligibility and oral health, and this approach should be considered for inclusion in clinical guidelines. However, longer term compliance and the potential impact of unknown differences in device durability are being explored in a longer term evaluation of this cohort to help determine whether minor differences between patient-fitted and dentally-produced MADs are important. In addition, the effects on cardiovascular events and road traffic accidents are being studied in a long-term cost-effectiveness model. Finally, whether adjustable devices are cost-effective and offer clinically significant advantages over bespoke non-adjustable devices in the real life setting still need to be explored.

Acknowledgements We are grateful to the TOMADO Trial Steering Committee (Professor John Gibson (Chair), Dr Justin Pepperell and Professor Mary Morrell), the Data Monitoring Committee (Dr Andrew Cummin (Chair), Dr Andrew Hall and Professor John Matthews) and the public patient involvement (PPI) representative,
Tanya Wanstall, for their contribution to the trial. We thank the staff of Meditas and the oral and maxillofacial surgery department at Addenbrooke's Hospital for making the devices. Thanks also go to the patients and staff in the Respiratory Support \& Sleep Centre at Papworth Hospital who made the trial possible, especially Clare East and Joanna Rayner. The protocol has been published in the Lancet Protocol Reviews (010PRT/4998).

Contributors TGQ conceived the study. TGQ, LDS, RC, MC and MJM designed the study. The systematic review of literature was written by MB, MJG, MAP and LDS. MAP, ALC-J and RC collected the data. MB, JJ, MJG and LDS analysed the data and all authors interpreted the data. TGQ, LDS and JAF-R drafted the manuscript. ALC-J edited the manuscript and all authors critically revised it.

Funding This project was funded by the National Institute for Health Research Health Technology Assessment (NIHR HTA) Programme (project number 08/110/03) and will be published in full in the Journal of Health Technology Assessment.

Competing interests $M C$ provided the bespoke mandibular advancement device service at Addenbrooke's Hospital, but has no personal or financial interests in the device. The manufacturer of the SP1 and SP2 supplied them at a discounted price but were not involved in the study design, analysis or reporting of results, nor did they have exclusive access to any part of the potential study data in return for the discount. As such, there are no conflicts of interest. LDS was partly funded by the Medical Research Council programme number U015232027. MB was funded by an NIHR Clinical Trials Methodology fellowship.

Ethics approval The National Research Ethics Service (NRES) Committee East of England - Cambridge Central (formerly known as Cambridgeshire 2 Research Ethics (ommittee) approved the protocol and subsequent amendments. The trial was registered with the International Standard Randomised Controlled Trial Number Register before the trial began (ISRCTN02309506).

Provenance and peer review Not commissioned; externally peer reviewed.

Data sharing statement This work was funded by the NIHR and all data will be published in full in the form of a single whole issue of Journal of Health Technology Assessment within the NIHR Journals Library (full draft report submitted in December 2013 for peer review and subsequent publication; no expected publication date yet).

\section{REFERENCES}

1 Douglas NJ, Polo 0. Pathogenesis of obstructive sleep apnoea/hypopnoea syndrome. Lancet 1994;344:653-5.

2 Punjabi NM. The epidemiology of adult obstructive sleep apnea. Proc Am Thorac Soc 2008;5:136-43.

3 Stradling JR, Pepperell JC, Davies RJ. Sleep apnoea and hypertension: proof at last? Thorax 2001:56(Suppl 2):ii45-9.

4 Dong JY, Zhang YH, Qin LQ. Obstructive sleep apnea and cardiovascular risk: meta-analysis of prospective cohort studies. Atherosclerosis 2013;229:489-95.

5 Redline S, Yenokyan G, Gottlieb DJ, et al. Obstructive sleep apnea-hypopnea and incident stroke: the sleep heart health study. Am J Respir Crit Care Med 2010;182:269-77.

6 Ellen RL, Marshall SC, Palayew M, et al. Systematic review of motor vehicle crash risk in persons with sleep apnea. J Clin Sleep Med 2006;2:193-200.

7 Moyer CA, Sonnad SS, Garetz SL, et al. Quality of life in obstructive sleep apnea: a systematic review of the literature. Sleep Med 2001;2:477-91.

8 Tarasiuk A, Greenberg-Dotan S, Simon-Tuval T, et al. The effect of obstructive sleep apnea on morbidity and health care utilization of middle-aged and older adults. J Am Geriatr Soc 2008;56:247-54.

9 Barnes M, McEvoy RD, Banks S, et al. Efficacy of positive airway pressure and oral appliance in mild to moderate obstructive sleep apnea. Am J Respir Crit Care Med 2004; 170:656-64.

10 Bazzano LA, Khan Z, Reynolds K, et al. Effect of nocturnal nasal continuous positive airway pressure on blood pressure in obstructive sleep apnea. Hypertension 2007:50:417-23.

11 Bradley TD, Floras JS. Obstructive sleep apnoea and its cardiovascular consequences. Lancet 2009:373:82-93.

12 National Institute for Health and Care Excellence. Continuous positive airway pressure for the treatment of obstructive sleep apnoea/hypopnoea syndrome. London: National Institute for Health and Care Excellence. TA139. http://www.nice. org.uk/nicemedia/live/11944/40085/40085.pdf

13 Weaver TE, Mancini C, Maislin G, et al. Continuous positive airway pressure treatment of sleepy patients with milder obstructive sleep apnea: results of the CPAP Apnea Trial North American Program (CATNAP) randomized clinical trial. Am J Respir Crit Care Med 2012;186:677-83.

14 Patel SR, White DP, Malhotra A, et al. Continuous positive airway pressure therapy for treating sleepiness in a diverse population with obstructive sleep apnea: results of a meta-analysis. Arch Intern Med 2003;163:565-71.

15 Weaver TE, Grunstein RR. Adherence to continuous positive airway pressure therapy: the challenge to effective treatment. Proc Am Thorac Soc 2008:5:173-8. 
16 Lim J, Lasserson TJ, Fleetham J, et al. Oral appliances for obstructive sleep apnoea. Cochrane Database Syst Rev 2006:CD004435.

17 Phillips CL, Grunstein RR, Darendeliler MA, et al. Health Outcomes of Continuous Positive Airway Pressure versus Oral Appliance Treatment for Obstructive Sleep Apnea. Am J Respir Crit Care Med 2013;187:879-87.

18 Petri N, Svanholt P, Solow B, et al. Mandibular advancement appliance for obstructive sleep apnoea: results of a randomised placebo controlled trial using parallel group design. J Sleep Res 2008;17:221-9.

19 McDaid C, Griffin S, Weatherly H, et al. Continuous positive airway pressure devices for the treatment of obstructive sleep apnoea-hypopnoea syndrome: a systematic review and economic analysis. Health Technol Assess 2009;13:iii-iv, xi-xiv, 1-119, 43-274.

20 Department of Health. NHS Reference costs (2011/12): Published 8 November 2012. https://www.gov.uk/government/publications/nhs-reference-costs-financialyear-2011-to-12

21 Personal Social Services Research Unit (PSSRU). Unit Costs of Health and Social Care (2012). http://www.pssru.ac.uk/project-pages/unit-costs/2012/index.php

22 Dolan P, Gudex C, Kind P, et al. A social tariff for EuroQol: results from a UK general population survey University of York. Center for Health Economics, 1995:1-24.

23 Sutherland K, Deane SA, Chan AS, et al. Comparative effects of two oral appliances on upper airway structure in obstructive sleep apnea. Sleep 2011;34:469-77.

24 Mehta A, Qian J, Petocz P, et al. A randomized, controlled study of a mandibular advancement splint for obstructive sleep apnea. Am J Respir Crit Care Med 2001;163:1457-61.

25 Vanderveken OM, Devolder A, Marklund $\mathrm{M}$, et al. Comparison of a custom-made and a thermoplastic oral appliance for the treatment of mild sleep apnea. Am J Respir Crit Care Med 2008;178:197-202.
26 Gotsopoulos H, Chen C, Qian J, et al. Oral appliance therapy improves symptoms in obstructive sleep apnea: a randomized, controlled trial. Am J Respir Crit Care Med 2002;166:743-8.

27 de Almeida FR, Bittencourt LR, de Almeida $\mathrm{Cl}$, et al. Effects of mandibular posture on obstructive sleep apnea severity and the temporomandibular joint in patients fitted with an oral appliance. Sleep 2002;25:507-13.

28 Andren A, Hedberg P, Walker-Engstrom ML, et al. Effects of treatment with oral appliance on 24-h blood pressure in patients with obstructive sleep apnea and hypertension: a randomized clinical trial. Sleep Breath 2013;17:705-12.

29 Aarab G, Lobbezoo F, Hamburger HL, et al. Oral appliance therapy versus nasal continuous positive airway pressure in obstructive sleep apnea: a randomized, placebo-controlled trial. Respiration 2011:81:411-19.

30 Tegelberg A, Walker-Engstrom ML, Vestling 0 , et al. Two different degrees of mandibular advancement with a dental appliance in treatment of patients with mild to moderate obstructive sleep apnea. Acta Odontol Scand 2003;61:356-62.

31 Crawford MR, Bartlett DJ, Coughlin SR, et al. The effect of continuous positive airway pressure usage on sleepiness in obstructive sleep apnoea: real effects or expectation of benefit? Thorax 2012;67:920-4.

32 Vanderveken OM, Dieltjens $\mathrm{M}$, Wouters $\mathrm{K}$, et al. Objective measurement of compliance during oral appliance therapy for sleep-disordered breathing. Thorax 2013;68:91-6.

33 Marklund M, Verbraecken J, Randerath W. Non-CPAP therapies in obstructive sleep apnoea: mandibular advancement device therapy. Eur Respir J 2012;39:1241-7.

34 Lettieri $\mathrm{CJ}$, Paolino $\mathrm{N}$, Eliasson $\mathrm{AH}$, et al. Comparison of adjustable and fixed ora appliances for the treatment of obstructive sleep apnea. J Clin Sleep Med 2011;7:439-45.

35 Schmidt-Nowara W. Recent Developments in Oral Appliance Therapy of Sleep Disordered Breathing. Sleep Breath 1999;3:103-06. 